\title{
The Effect of Reading Strategies and Prior Knowledge on Cognitive Load and Learning with Hypertext
}

\author{
R. Ignacio Madrid* and José J. Cañas
}

\author{
Cognitive Ergonomics Group, Department of Experimental Psychology, University of Granada, Campus de Cartuja, s/n. \\ 18071 Granada, Spain
}

\begin{abstract}
Reading strategies, prior knowledge and cognitive load are some variables that have been related with comprehension and learning with hypertext systems. In this study we analyze the effect of two different hypertext reading strategies - coherence and interest - and two prior knowledge levels - low and high - on cognitive load, and their relation with learning. For low prior knowledge readers, data reveal that following a coherence strategy leads to lower cognitive load during reading and better learning. For high prior knowledge readers, following an interest strategy produce higher cognitive load during reading than a coherence strategy, but they learned equally from both strategies. These results are discussed taking into account the implication of two different components of cognitive load (extraneous and germane cognitive load).
\end{abstract}

\section{INTRODUCTION}

Hypertext is currently widely used as an essential component in formal learning (e.g. higher education elearning systems) as well as informal or incidental learning (e.g. Wikipedia). Hypertexts have the advantage of fostering learner control, which is performed through sequencing (the order in which the learner want to access the different information units) and selection or content control (which contents to read from a set of documents) [1]. The specific rule that a hypertext user follows for sequencing and content control are known as reading strategies [2,3].

Fostering learner control should lead to better motivation, learner engagement and, subsequently, a better learning. However, in a recent review on learner control and hypermedia learning [1] the authors have claimed that the effectiveness for learning of learner control has been shown not to be general and it could be affected by both system and learner characteristics. For example, there is summative evidence showing that low prior knowledge learners have problems with hypertext, and it may be more suited for expert learners [4].

Additionally, the authors also reviewed studies that suggest that cognitive resources needed for learner control will not be available for learning, and if they are high it can lead to cognitive overhead and impairment in learning. Therefore, the question is under which circumstances, learner control leads to high cognitive load and impairment on learning.

In this study, we will examine the effect of two different reading strategies - used by both low and high prior knowledge learners - on cognitive load and learning.

*Address correspondence to this author at the Cognitive Ergonomics Group, Department of Experimental Psychology, University of Granada, Campus de Cartuja, s/n. 18071 Granada, Spain;

E-mail: nmadrid@ugr.es

\section{READING STRATEGIES AND PRIOR KNOWLEDGE}

The C-I model of text comprehension [5-7] consider text coherence and prior knowledge as the main variables affecting text comprehension. Text coherence is a complex construct that depends on several factors, but it can be defined in a simple way as the extent to which a reader can understand the relations between ideas expressed in a text [8]. In some linear text reading studies it has been shown a learning reverse effect [9-11]. Low prior knowledge (LPK) readers find high coherent texts beneficial since they don't have the necessary background knowledge to infer information that is not directly stated in the text. However, high prior knowledge (HPK) readers learn more with a less coherent text. The explanation of this reverse effect is that HPK readers are less likely to use their prior knowledge if the text is highly coherent, and reading a less coherent text activates their prior knowledge through inferential processes leading to better learning.

In hypertext comprehension studies, text coherence has been also shown to be important for learning, with the difference that it can be modified through the reading strategies that readers follows $[2,3,12]$. Reading strategies are general navigation rules that users follow to select what hypertext contents to read (selection or content control) and in which order (sequencing). Several strategies have been examined, but the main ones seem to be coherence, interest and link location $[3,13]$.

In two experiments, Salmerón, Kinstch \& Cañas [3] examined the effects on comprehension of different criteria for selecting links, being the main ones coherence and interest:

a) Coherence strategy consists in selecting the link most related with the text just read, and therefore it promotes high reading text coherence. As stated before, reading text coherence in hypertext depends 
also on learners' control, since the reading order selected by the readers determines the coherence of the reading sequence [12].

b) Interest strategy is based in selecting first the links that seems most interesting to the reader, delaying the reading of the less interesting ones. Therefore the interest strategy should produce a less coherent reading sequence than the coherence strategy.

The results of the experiments conducted [3] showed that readers following these different reading strategies obtained different comprehension outcomes: LPK readers achieved better comprehension with the coherence strategy than with the interest strategy, but readers with higher knowledge comprehended the contents equally well using the coherence or the interest strategy. Moreover, the results suggested that the effects of reading strategies on comprehension are achieved through two different mechanisms. The first is textinduced, as in linear text reading a high-coherence text is better for LPK readers than reading a low-coherence one. On the other hand, readers with some knowledge can obtain benefits from reading low-coherence texts since this process helps them to avoid the shallow processing caused by highcoherence texts $[9,14]$. The second mechanism is a strategic influence: for readers with some prior knowledge following a strategy to select the reading order activates their prior knowledge and automatically mobilizes cognitive resources for learning. On the contrary, LPK readers did not get benefits from the strategic influence and they don't achieve better learning compared with reading a linear text with the same coherence level. In summary, HPK are affected both by the text-induced and the strategic influence mechanism, and they learn equally both from the coherence or from the interest strategy, while LPK readers are affected only by the text-induced mechanism and they achieved a better learning when they used a coherence strategy.

\section{COGNITIVE LOAD DURING HYPERTEXT READING}

The Cognitive Load Theory (CLT) is directed to guide instructional design decisions based on the way in which cognitive resources are used during learning [15-17]. The theory distinguishes between three types of cognitive load $(C L)$ : intrinsic CL, extraneous CL and germane CL. Intrinsic CL is related with prior knowledge and the nature of the materials to be learnt (interactivity between elements). Extraneous CL (ineffective for learning) is the effort required by poorly designed tasks, while germane CL (effective for learning) concerns activities related with the construction of schemas and automation leading to higher levels of comprehension. For an instructional design (e.g. educational hypertext) to be effective for learning, extraneous CL have to be reduced and germane CL have to be enhanced.

In the context of hypertext reading, CL has been also analyzed to study their relation with learning $[18,19]$. From the point of view of the human cognitive system characteristics, hypertext navigation requires a large amount of cognitive resources. Cognitive resources are needed to plan navigation, to assess the relevance of the information found and to comprehend the information and to integrate it with prior knowledge. In a recent review, DeStefano \&
LeFevre [18] claimed that, compared with traditional printed text, hypertext tasks requires extra working memory resources to be allocated to decision and comprehension processes. This increment could lead to comprehension problems, mainly for low knowledge readers. They predicted that this CL increment comes from two sources: First, from the decision-making processes needed to perform navigation (and therefore, if more links are offered the CL would be higher); second, from the difficulty of reading and comprehending the information when links followed leads to information semantically unrelated with the previously read contents that can hinder the construction of situation models (and therefore, offering only links to closely related information would reduce $\mathrm{CL}$ ).

To test these prediction, Madrid, Van Oostendorp \& Puerta Melguizo [20], run an experiment in which different types of hypertext presentations ( 3 vs 8 links menus, showing or not link suggestions based on semantic relatedness) were used by LPK readers to test their CL level and learning. The participants were instructed to follow a coherence strategy, selecting the link that they thought that were more related with the previously read text. Participants had to read all the contents, and therefore learner control in this experiment is reduced to sequencing (they were not allowed to select what contents to read). The results showed that $\mathrm{CL}$ during hypertext reading and learning were mediated by the reading order that readers followed. Their achieved reading text coherence was measured, and two groups of low and high text coherence reading orders were constructed. Participants selecting a high text coherence reading order suffered less CL both during reading and during link selection, and achieved a better learning than those selecting a low text coherence reading order. This experiment only partially supported DeStefano \& LeFevre's [18] predictions, but confirmed the role of text coherence both in CL and learning, at least with LPK readers.

Moreover, the experiment [20] showed that readers' activities are more important for learning than hypertext design characteristics. In a similar way, Gerjets \& Scheiter [21] proposed an extension of the CLT based on the assumption that instructional design is not directly determining cognitive load, but that learning activities are important moderators between instructional design and cognitive load.

As have been showed before, readers' prior knowledge is a main variable affecting learning with hypertext systems. However, there is little research concerning the role of prior knowledge on CL during hypertext reading. An exception is the study carried out by Amadieu, Tricot \& Marinee [22] who conducted two experiments exploring the relation between prior knowledge, CL, navigation and learning. Although one of the experiments failed to find differences between low and high knowledge readers in CL, the results of the other experiment suggested that prior knowledge could have a negative relation with CL (i.e. the higher the prior knowledge, the lower the $\mathrm{CL}$ ).

Summarizing, the studies described above suggest that certain features of reading strategies (as reading text coherence, i.e. text-induced mechanism) and readers' prior knowledge determine $\mathrm{CL}$ and therefore they could affect learning. However, it is needed to notice that the 
relationships between $\mathrm{CL}$ and learning would be misunderstood if we don't take into account the nature of CL. In this sense, studies as [18] focused in the idea that the more CL invested in hypertext, the worse the performance. This should be only true if the CL exceeds the limits of working memory [23]. But, on the contrary, if CL remains within the limits of working memory, a higher investment of mental effort could also produce better learning when the extra resources are used for active processing [24].

\section{RESEARCH OBJECTIVES}

This study is an extension of the line of research started by Madrid, Van Oostendorp \& Puerta Melguizo [20]. Their experiment had two main limitations concerning the role of cognitive load in hypertext reading.

First, only low prior knowledge readers participated in the experiment, who following a previous study [3] are only influenced by the text-induced mechanism (i.e. there is not a strategic influence). Since readers who performed a less coherent reading order got higher CL and worse learning, we can reach the conclusion that the increase in CL was mainly composed of extraneous CL. However, for readers with some prior knowledge, following a less coherent reading order could lead to better learning. Moreover, the strategic influence mechanism could produce germane CL for HPK readers that are not available for LPK readers. Therefore, to test the hypothetical effects of the strategic influence mechanism on CL, new experiments are needed in which learners with higher prior knowledge are also included.

Second, to control for the effect of different strategies all the participants were instructed to follow the coherence strategy. However, trying to achieve high reading text coherence is not the only reading strategy that readers could perform, and following different reading strategies may have differential effects on CL as it does on learning [3]. This effect could be mainly relevant during link selection, since different reading strategies could need different amount of resources to assess the relevance of link labels for their goals, but also during reading, since different reading strategies could lead readers to focus in different aspects of the text. Supporting these ideas, evidence coming from the field of cognitive ergonomics shows that people that use alternative strategies for doing a task differ in the amount of mental effort needed for task performance $[25,26]$.

To solve these limitations, in this study we tested the effect of different reading strategies at different levels of prior knowledge on CL and learning. As in the experiment from Madrid, Van Oostendorp \& Puerta Melguizo [20], the participants read all the contents and they could only control sequencing. The aim of this experiment was two-fold:

1) The effect of reading strategies on CL: We examined the way in which two different reading strategies coherence and interest - could affect the CL that low prior knowledge (LPK) readers and high prior knowledge (HPK) readers experience with hypertext. For both LPK and HPK readers, we predicted that the coherence strategy would produce higher CL during link selection than the interest strategy, since performing the coherence strategy requires several semantic relatedness judgments to select the most related link, while the interest strategy only needs of personal preferences decisions which a priori would consume less cognitive resources. Conversely, we expected that both LPK and HPK readers following the interest strategy would experience higher levels of CL during reading - because of the lower reading text coherence achieved using this strategy - than those following the coherence strategy.

2) The relation between reading strategies, CL and learning: We tested the effect of reading strategies and prior knowledge on learning, and putting together with CL, we used the overall pattern of results to analyze the relation between CL and learning. According with Salmerón, Kintsch \& Cañas [3] and the Cognitive Load Theory [15], we expected that the increase on CL during reading for LPK readers using an interest strategy in relation with those using the coherence strategy would be mainly composed of extraneous CL, and therefore their learning would be lower. On the other hand, for HPK readers both strategies could be good for learning: Using the interest strategy they could obtain reading orders with lower text coherence and a higher overall CL, but they could transform the extra processing into germane CL during reading. Additionally, through the strategic influence mechanism, HPK readers could invest extra germane CL if they follow a coherence strategy, and therefore they could avoid the shallow processing induced by high coherence texts.

\section{METHOD}

\section{Participants}

Fifty-two students of the University of Granada participated in the experiment. Half of them were secondstage Psychology students (at least 3 years studying Psychology), and the other half were Psychology freshmen (less than one semester studying Psychology) or students from other disciplines (Education, Language or Sport Sciences) with only an introductory course on Psychology. They received course credits for their participation.

\section{Design}

The study followed an experimental $2 \times 2$ design with type of reading strategy (coherence $v s$ interest) and prior knowledge level (low vs high prior knowledge) as independent variables. We used two kinds of dependent variables: reading processes measures and learning measures. As reading processes measures we used reading text coherence (mean LSA cosines between text transitions), average reaction times to a secondary task (both when reading texts and when selecting links) and reading and link selection times. As a learning measure we used the score on an inference questionnaire.

\section{Materials}

Participants read a text in Spanish about Neuropsychology, extracted from a General Psychology introductory ebook. The text had 4599 words and was divided into 21 pages.

After reading each text page, readers went to the link selection menu, where they had to select the next text to read within 8 links. The menu was constructed by showing the 
two most related links with the text just read, and six links more that were extracted randomly from the list of link labels.

Participants had to select the next text to read following the specific instructions for their type of strategy condition. Participants in the coherence condition had to select the link that they assessed as the most similar to the text just read. Readers that were assigned to the interest condition had to select the link that they assessed as the most interesting to them.

\section{Measures}

\section{Process Measures}

Reading Text Coherence: Based on the reading order followed by the participant, we computed the mean LSA cosine of their text transitions (see [12] for a detailed explanation of this method). This measure can be used as an index of the coherence of the reading order followed by the readers $[3,12,20]$.

RTs to Secondary Task: This technique has been widely used to measure the $\mathrm{CL}$ associated with different experimental treatments [27-30]. It requires participants to perform the main task or primary task while responding to random beeps as quickly as possible (secondary task). RTs to beeps are slower when the cognitive requirements of the primary task are higher, and therefore can be used to measure the $\mathrm{CL}$ associated with it. This measure has showed sensitive to extraneous $\mathrm{CL}$ and to inefficient learning [20, $31,32]$.

In our experiment, at the beginning of the session participants had to react as quickly as possible to 10 beep sounds presented randomly to obtain their RT baseline. During hypertext reading, participants had to press the " $\mathrm{z}$ " key as soon as possible when a beep was presented through the headphones. Their data was corrected by subtracting the baseline RTs. Variations in RTs reveal the cognitive capacity allocated to the primary tasks: reading or selecting links. Consequently, we computed the corrected RTs separately when selecting links and when reading the text fragments.

Several measures of cognitive load derived from RTs can be computed [23]. In our analyses, we will use the average RTs both for reading and selecting links which reflects the intensity of the cognitive load carried out during the task. However, some authors have claimed that not only the intensity but also the duration of the CL is relevant [33, 34].
Reading and Link Selection Times: To cope with the duration aspects of CL, the time spent when reading and when selecting links was measured and analyzed separately. Time has been used to test the cognitive load required both in reading [35] and in menu navigation tasks [36]. Link selection times were recorded in seconds for each page, starting when the link menu was shown and finishing when a link label was clicked. Reading times were measured in seconds for each hypertext page. A total reading and total link selection time was computed for each subject by adding the times spent for each task in each page.

\section{Learning Measure}

Inference questions score: Several mental representations are constructed in the process of learning from text [37]. The situation model is considered the deepest mental representation, formed when the textbase propositions are integrated with prior knowledge. It this experiment, a questionnaire composed of ten inference questions was administered to all participants after they completed the hypertext reading task. It was constructed in such manner that the questions and the answers appeared in different hypertext pages. The score in this questionnaire was used as a measure of situation model acquisition. Chance performance was at $25 \%$.

\section{RESULTS}

All results were considered significant when $\mathrm{p}<.05$, and marginally significant when $p$ values were between .05 and .10 . The data of one participant was excluded from the analyses as extreme outlier. Therefore the following results are based in a sample of 51 participants. According with Salmerón, Kintsch \& Cañas [3] and Cognitive Load Theory [15].

\section{Process Measures: Reading Text Coherence, Cognitive Load and Reading and Link Selection Times}

A set of $2 \times 2$ ANOVAs was performed using prior knowledge and type of strategy as independent variables, and reading text coherence, mean RTs and reading and link selection times as dependent variables. See Table 1 for a summary of mean and SD results per group.

\section{Reading Text Coherence}

Results showed a main effect of type of strategy $(\mathrm{F}(1,51)$ $=48.177 ; \mathrm{p}<.001)$. Participants in the coherence condition selected a more coherent reading order (higher mean LSA cosine, $\mathrm{M}=0.317$; $\mathrm{SD}=0.047)$ than those in the interest strategy $(\mathrm{M}=0.211 ; \mathrm{SD}=0.011)$. There were not significant differences for prior knowledge, and no interaction effect.

Table 1. Mean and SDs (Between Parentheses) on Reading Processes Measures

\begin{tabular}{|l|c|c|c|c|}
\hline \multirow{2}{*}{} & \multicolumn{2}{|c|}{ Low Knowledge } & \multicolumn{2}{|c|}{ High Knowledge } \\
\cline { 2 - 5 } & Coherence & Interest & Coherence & $0.324(0.046)$ \\
\hline \hline LSA cosines & $0.309(0.049)$ & $0.206(0.059)$ & $198(82.4)$ & $0.216(0.068)$ \\
\hline Average reaction times (link selection) & $202(61.5)$ & $216(105.7)$ & $151(59.4)$ & $276(73.8)$ \\
\hline Average reaction times (reading) & $150(29.7)$ & $135(68.6)$ & $225.14(90.20)$ & $140(68.5)$ \\
\hline Link selection times & $293.77(127.74)$ & $162.86(65.88)$ & $1199.429(300.47)$ & $1340.661(419.08)$ \\
\hline Reading times & $1561.92(361.33)$ & $1783.35(306.01)$ & $(60.08)$ \\
\hline
\end{tabular}




\section{Reaction Times to Secondary Task}

Average reaction times during reading: Contrary to our expectations, the $2 \times 2$ ANOVA results on mean reaction times during reading did not reach statistical significance (all p's $>0.1)$.

Average reaction times during link selection: Results showed a significant main effect of type of strategy $(F(1$, $51)=4.25 ; \mathrm{p}<.05)$. Readers using the interest strategy got higher reaction times $(M=246 ; S D=94)$ than those using the coherence strategy $(M=200 ; \mathrm{SD}=72)$. There were not main effects of prior knowledge or interaction effects.

Fig. (1) shows the results both for link selection and reading.

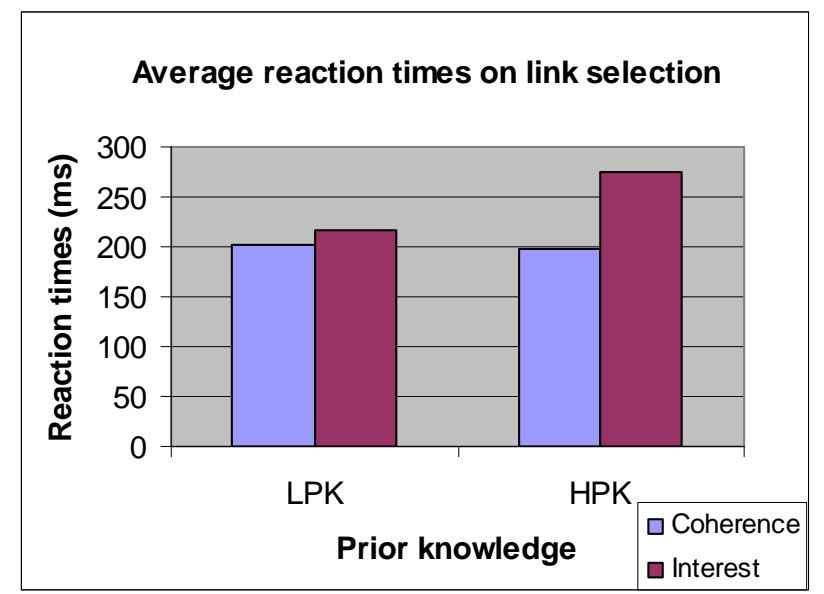

186.50; $\mathrm{SD}=16.84)$. The interaction did not reach significance $(p>1)$.

Reading times: Results showed a main effect of prior knowledge $(F(1,51)=18.19 ; \mathrm{p}<.001)$, LPK readers devoted more time to reading $(\mathrm{M}=1672.64 ; \mathrm{SD}=67.37)$ than HPK readers $(\mathrm{M}=1270.05 ; \mathrm{SD}=66.11)$. The analysis also showed a marginally significant effect of type of strategy $(F(1,51)=3.69 ; p=0.06)$, the interest strategy group devoted more time to reading $(\mathrm{M}=1562 ; \mathrm{SD}=66.11)$ than the coherence strategy group $(\mathrm{M}=1380.68 ; \mathrm{SD}=67.38)$. The interaction did not reach significance $(\mathrm{F}<1)$.

Fig. (1). Average reaction times during reading and during link selection.

\section{Reading and Link Selection Times}

Fig. (2) show the graph for results both on link selection and reading times.

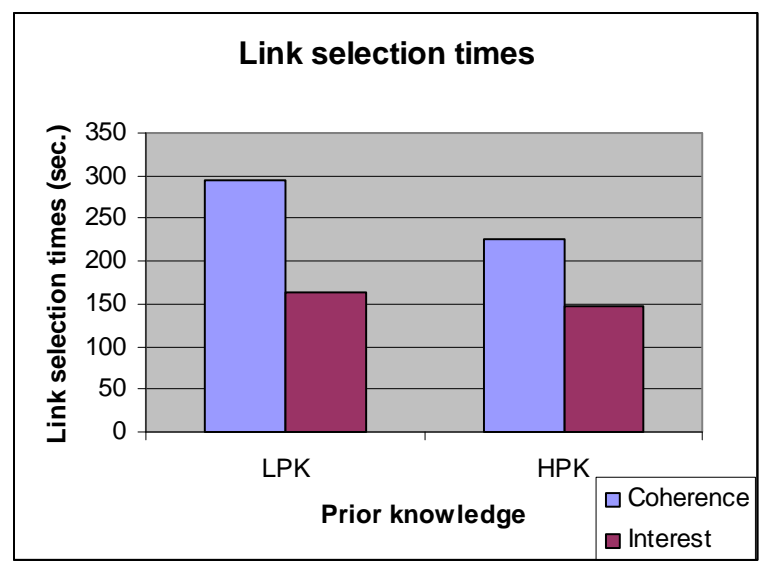

Fig. (2). Link selection times and reading times.

Link selection times: Time devoted to link selection was higher for those using the coherence strategy $(\mathrm{M}=259.46$; $\mathrm{SD}=17.16)$ than for those using the interest strategy $(\mathrm{M}=$ 155.36; $\mathrm{SD}=16.84),(\mathrm{F}(1,51)=18.75 ; \mathrm{p}<.001)$. A main effect of PK was found marginally significant $(F(1,51)=$ $3.02 ; \mathrm{p}<.09$ ), LPK readers seems to expend more time selecting links $(\mathrm{M}=228.31 ; \mathrm{SD}=17.16)$ than $\mathrm{HPK}(\mathrm{M}=$
Learning Measure: Score on Inference Questions Questionnaire

A $2 \times 2$ ANOVA was performed using prior knowledge and type of strategy as independent variables, and inference

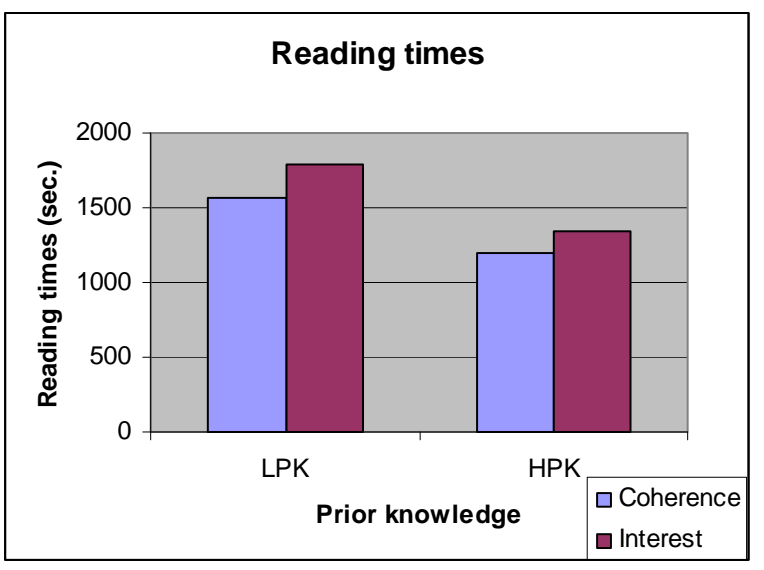

questions score as dependent variable. Mean and standard deviation are showed in Table 2. See also Fig. (3) for a graphical representation.

\section{Inference Questions}

Results showed a main effect of prior knowledge $(\mathrm{F}(1,51)=$ $13.70, \mathrm{p}=.001)$, and a significant interaction effect $(\mathrm{F}(1,51)=$ $5.14 ; \mathrm{p}<.05$ ) (See Table 2 and Fig. 3). Pairwise comparisons 
showed that LPK readers acquired a better situation model when using the coherence strategy (near significant, $\mathrm{F}(1,51)=$ $3.82 ; \mathrm{p}=0.56$ ), but no differences were found for HPK. Comparisons also showed that HPK readers got better situation models than LPK readers when they used an interest strategy $(\mathrm{F}(1,51)=18.16 ; \mathrm{p}<.001)$, but there learnt equally when they use the coherence strategy $(\mathrm{F}<1)$.

Table 2. Average RTs Per Condition (Standard Deviations Between Parentheses)

\begin{tabular}{|c|c|c|c|c|}
\hline \multirow{2}{*}{} & \multicolumn{2}{|c|}{ Low Knowledge } & \multicolumn{2}{c|}{ High Knowledge } \\
\cline { 2 - 5 } & Coherence & Interest & Coherence & Interest \\
\hline \hline $\begin{array}{l}\text { Inference } \\
\text { questions }\end{array}$ & $4.15(1.91)$ & $2.79(1.37)$ & $4.86(1.99)$ & $5.71(1.94)$ \\
\hline
\end{tabular}

\section{DISCUSSION AND CONCLUSIONS}

The main objective of this experiment was to analyze the effect of reading strategies and prior knowledge on cognitive load, and their relation with learning.

First of all, we assumed that the interest reading strategy would lead to a less coherent reading order than the coherence strategy. Confirming that assumption, mean LSA cosines were quite lower for those following the interest strategy.

\section{The Effect of Reading Strategies and Prior Knowledge on Cognitive Load}

We predicted that following a coherence strategy would result in higher CL during link selection both for LPK and HPK readers. This assumption relays in the cognitive processes underlying link selection, since interest is an "a priori" automatic and motivational process while coherence requires several semantic similarity comparisons to be performed which would consume cognitive resources. Contrary to our expectations, the intensity of the CL (measured with RTs to secondary task) was higher for the interest strategy. In addition, we expected that both LPK and
HPK readers using the interest strategy would experience higher CL during reading than those using the coherence strategy. However, both groups experienced the same intensity of CL. This last prediction was based on the study from Madrid, Van Oostendorp \& Puerta Melguizo [20] that showed that those LPK readers following a reading order with lower coherence got higher intensity of CL. Although the reading text coherence for the interest strategy group was considerably lower than for the coherence strategy group their mean RTs were similar.

These results could seem difficult to reconcile with our hypotheses. However, before discussing results on the intensity of the CL we have to take into account also the duration on the CL. The notion of "volume of attention" can be relevant here: both the intensity and duration of the CL are important for learning [38, 39]. The "volume of attention" hypothesis shows the dynamics of CL: task A can be more cognitive demanding than task $\mathrm{B}$, but it can be performed using the same intensity of CL if more time is devoted to it. Therefore, to discuss our results we also have to take into account reading and link selection times.

On the one hand, analyses of link selection times reflected that the time requirements are higher for the coherence strategy than for the interest strategy, both for LPK and HPK readers. Therefore, those readers following the coherence strategy showed higher duration but lower intensity of CL than the interest group. This pattern of CL results prevents us to make any conclusion about which strategy required higher CL, although it can be interpreted as an evidence for a different tradeoff between intensity and duration for both strategies.

On the other hand, the analyses of reading times showed that the interest strategy required more time than the coherence strategy both for LPK and HPK. Since no differences were found on the intensity of CL, it can be concluded that higher reading and link selection times mean higher cognitive requirements for the interest condition. Conversely, in the experiment from Madrid, Van Oostendorp \& Puerta Melguizo [20], higher reading text coherence led to

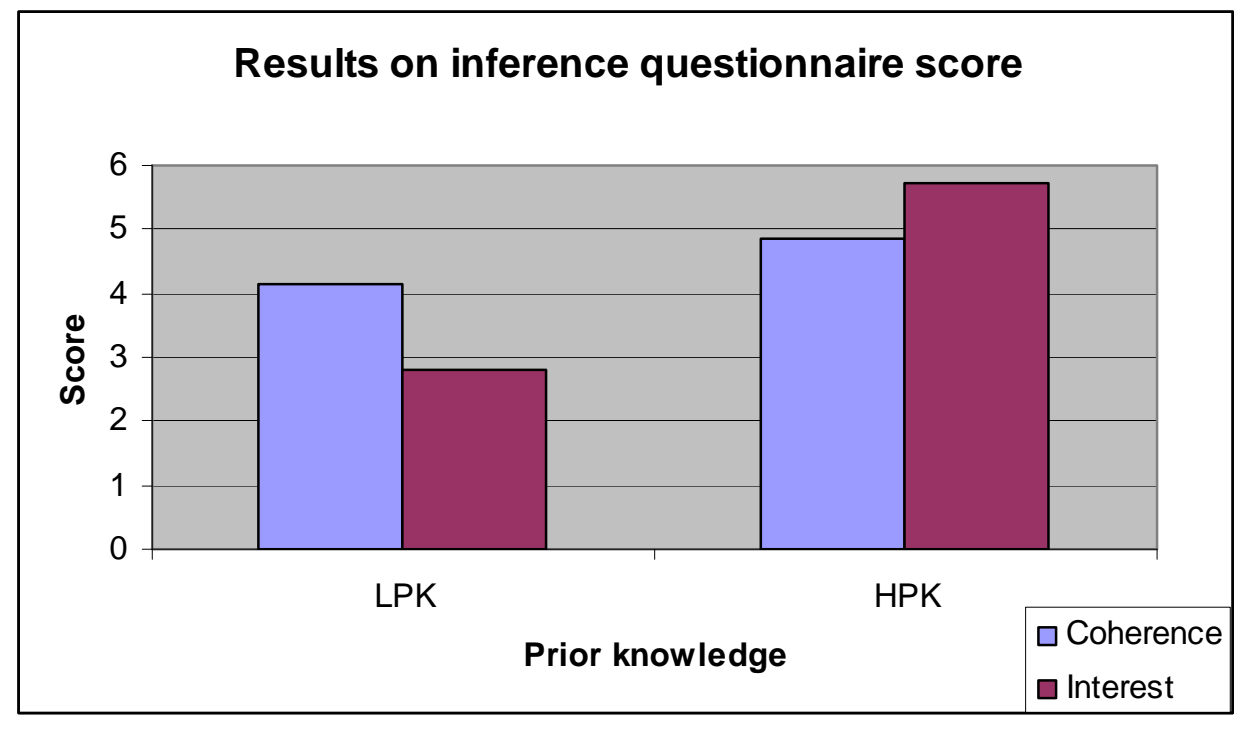

Fig. (3). Inference questions score per condition. 
higher intensity of CL (average RTs) but no differences were found on reading times.

As a summary of this section, it can be argued that our prediction on the role of reading strategies on CL is partially supported. Both LPK and HPK readers who followed the interest strategy had higher CL during reading than those who followed the coherence strategy. Regarding the effect of reading strategies on link selection, those who followed a coherence strategy had not higher requirement than those who followed an interest strategy, but they showed a different tradeoff between intensity and duration of CL.

\section{The Effect of Reading Strategies and Prior Knowledge on Learning}

In general, learning outcomes confirmed our predictions. Learning outcomes has been shown to be affected by the strategy used, and to be different for both LPK and HPK readers. The score on inference questions was higher for LPK readers that used the coherence strategy than for those that used the interest strategy. Otherwise, HPK readers learn equally either using the interest or the coherence strategy.

These results also support the widespread idea which argues that reading hypertext in a semantically unrelated order is harmful for LPK readers' learning [3, 18, 20, 40].

\section{The Relation Between $C L$ and Learning}

As this pattern of results shows, the relation between cognitive load and hypertext reading are far from being simple. Following the Cognitive Load Theory [15], there are three components of cognitive load that are additive (intrinsic, extraneous and germane cognitive load). Intrinsic cognitive load depends on the complexity of the materials and learner expertise. Extraneous cognitive load is related with inefficient learning. Finally, germane cognitive load are related with deeper learning. This distinction between different dimensions of CL is very useful from a theoretical point of view, but it has some methodological problems. First of all, experimental manipulations can influence more than one type of cognitive load at the same time, and it could have different effects on learning for LPK and HPK learners [41]. Second, the techniques used to measure CL do not clearly distinguish between these load components since they offer a global measure of CL [34]. If different measures were sensitive to different CL components, we could predict learning results based on the balance between intrinsic, extraneous and germane CL.

Recently, DeLeeuw \& Mayer [32] have discussed some research findings in the field of multimedia learning suggesting that different measures of CL (RT to secondary task, mental effort during learning and difficulty ratings) could be sensitive to different types of CL. In two experiments, participants (mainly low knowledge learners) watched different versions of a 6-minutes multimedia lesson, in which the three measures of CL were obtained. Results showed that RT to secondary task was mainly sensitive to manipulations in extraneous CL, whereas mental effort and difficulty ratings were sensitive to intrinsic and germane $\mathrm{CL}$ respectively. Regarding extraneous $\mathrm{CL}$, the measurement method (RT to secondary task), participant characteristics (low prior knowledge) and task timing features (no differences between conditions in relation to duration of the session) were similar to those in the Madrid, Van Oostendorp \& Puerta Melguizo experiment [20] in which the group with higher RTs also achieved less learning. Comparisons with the current experiment are difficult since participants had different levels of prior knowledge and we used also reading and links selection times as measures of $\mathrm{CL}$, which a posteriori appeared as more sensitive to complexity variations than RTs. Unfortunately, the duration of the session in the DeLeeuw \& Mayer experiment [32] was kept constant for all participants and therefore the results did not offer information on which component of CL could be related with performance time. Additionally, the authors pointed out some limitations on their study and they proposed replication with different learners and materials.

In spite of this lack of distinctive CL measures, in order to explore how reading strategies, CL and learning are related, the pattern of results on CL measures and learning measures can be analyzed. However, in this discussion we will exclude CL during link selection, focusing in CL during reading. The main reason to do this is that we did not obtain a clear measure of CL during link selection that could be used for the comparison, but it can be also argued that reading is the main task for learning with hypertext and therefore the effect of CL during link selection on learning could be overshadowed by the influence of CL during reading.

In line with prior research $[18,20]$, low knowledge participants that read the hypertext in a high coherent order by following a coherence strategy - got lower cognitive load when reading and better comprehension than those in the interest condition (who read the hypertext in a low coherence order). This is a support for the idea that low reading text coherence leads to extraneous CL, at least with LPK readers. Conversely, the pattern of results on learning and cognitive load when reading for HPK readers was different: those participants in the interest condition (who performed a less coherent reading order) got higher cognitive load than those in the coherence condition, but they achieved the same learning outcomes than those using the coherence strategy. Contrary to LPK readers, HPK readers can get higher germane $\mathrm{CL}$ with the interest strategy by activating prior knowledge and investing extra cognitive resources in learning. Moreover, the coherence strategy leads to follow a high coherence reading order which would hamper the activation of prior knowledge and would lead to extraneous CL for HPK readers, but the active selection of the reading order in the coherence strategy helps them to avoid the shallow processing induced by high coherent texts. Therefore, it seems that for HPK readers there are a balance in the coherence condition between extraneous $\mathrm{CL}$ and germane CL, and a higher investment of germane CL in the interest strategy. This balance of CL components helps them to learn equally from both strategies.

\section{Practical Implications}

In the preceding sections we have argued that using different reading strategies affect differently cognitive load, and subsequently learning with hypertext. Thus, by determining which strategy is better for a certain level of prior knowledge it is possible to match readers and strategies 
to enhance learning. Following this argument we can draw two practical implications of this experiment.

First, novices learn better when they follow a strategy that maximizes text coherence. Indeed, some authors argue that, to maximize learning, linear text is a better instructional design than hypertext for low prior knowledge readers [18]. However, maximize learning could be not the only reason to use hypertexts instead of linear text, and ease of access, interactivity, teacher control or economic factors could also recommend the use of an educational hypertext. Under these situations, it is important to support the learner in reading the materials in a coherent manner. In this sense, McNamara \& Shapiro [42] have proposed that it can be done with reading strategy training or including hypertext design features for user support.

Second, both low prior knowledge and high prior knowledge readers requires more time to perform an interest strategy. However, this increment is not related with a benefit in learning compared with the coherence strategy. Therefore, under instructional conditions in which duration is an important factor (e.g. educational programs defined on an hourly basis), to select a coherence strategy will be more time-saving.

\section{REFERENCES}

[1] Scheiter K, Gerjets P. Learner control in hypermedia environments. Educ Psychol Rev 2007; 19(3): 285-307.

[2] Madrid RI, Cañas JJ. How reading strategies affect the comprehension of texts in hypertext systems. In: Alamargot D, Terrier P, Cellier JM, Eds. Written documents in the workplace. Amsterdam: Elsevier 2007; pp. 205-15.

[3] Salmerón L, Kintsch W, Cañas JJ. Reading strategies and prior knowledge in learning with hypertext. Mem Cogn 2006; 34(5): 1157-71.

[4] Chen SY, Fan JP, Macredie RD. Navigation in hypermedia learning systems: Experts vs novices. Comput Hum Behav 2006; 22: 251-66.

[5] Kintsch W. The role of knowledge in discourse comprehension: A construction-integration model. Psychol Rev 1988; 95: 163-82.

[6] Kintsch W. Comprehension: A paradigm for cognition. New York: Cambridge University Press 1998.

[7] Van Dijk TA, Kintsch W. Strategies of discourse comprehension. New York: Academic Press 1983.

[8] Britton B, Gülgöz S. Using Kintsch's computational model to improve instructional text: Effects of inference calls on recall and cognitive structures. J Educ Psychol 1991; 83: 329-45.

[9] McNamara DS. Reading both high-coherence and low coherence texts: Effects of text sequence and prior knowledge. Can J Expl Psychol 2001; 55: 51-62.

[10] McNamara DS, Kintsch E, Songer N, Kintsch W. Are good texts always better? Interaction of text coherence, background knowledge, and levels of understanding in learning from text. Cognit Instruct 1996; 14: 1-42.

[11] McNamara DS, Kintsch W. Learning from text: Effect of prior knowledge and text coherence. Discourse Process 1996; 22: 24788.

[12] Salmerón L, Cañas JJ, Kintsch W, Fajardo I. Reading strategies and hypertext comprehension. Discourse Process 2005; 40(3): 171-91.

[13] Protopsaltis A. Reading strategies in hypertexts and factors influencing hyperlink selection. J Educ Multi Hyper 2008; 17(2): 191-213.
[14] Kintsch E, Kintsch W. Strategies to promote active learning from text: Individual differences in background knowledge. Swiss J Psychol 1995; 54: 141-51.

[15] Paas F, Renkl A, Sweller J. Cognitive load theory instructional implications of the interaction between information structures and cognitive architecture. Instr Sci 2004; 32: 1-8.

[16] Sweller J. Cognitive load theory, learning difficulty and instructional design. Learn Instr 1994; 4: 295-312.

[17] Sweller J, van Merriënboer JG, Paas F. Cognitive architecture and instructional design. Educ Psychol Rev 1998; 10(3): 251-96.

[18] DeStefano D, LeFevre JA. Cognitive load in hypertext reading: A review. Comput Hum Behav 2007; 23(3): 1616-41.

[19] Zumbach J, Mohraz M. Cognitive load in hypermedia reading comprehension: Influence of text type and linearity. Comput Hum Behav 2008; 24(3): 875-87.

[20] Madrid RI, Van Oostendorp H, Puerta Melguizo MC. The effects of the number of links and navigation support on cognitive load and learning with hypertext: The mediating role of reading order. Comput Hum Behav 2009; 25(1): 66-75.

[21] Gerjets P, Scheiter K. Goal configurations and processing strategies as moderators between instructional design and cognitive load: Evidence from hypertext-based instruction. Educ Psychol 2003; 38: 33-41.

[22] Amadieu F, Tricot A, Marinee C. Effects of prior domain knowledge on comprehension of non-linear documents: Proceedings of the Workshop on Cognition and the Web; April 2426, 2008. Granada, Spain.

[23] Xie B, Salvendy G. Prediction of mental workload in single and multiple task environments. Int J Cogn Ergonom 2000; 4: 213-42.

[24] Zumbach J. Cognitive overhead in hypertext learning re-examined: Overcoming the myths. J Educ Multi Hyper 2006; 15(4): 411-32.

[25] Brainbridge L. Problems in the assessment of mental load. Le Trav Hum 1974; 37(2): 279-302.

[26] Brainbridge L. Processes underlying human performance. In: Garland DJ, Wise JA, Hopkin VD, Eds. Handbook of Aviation Human Factors. Mahwah, NJ: Lawrence Erlbaum Associates; 1998; pp.107-71.

[27] Bonnardel N, Piolat A. Design activities: hot to analyze cognitive effort associated to cognitive treatments? Int J Cogn Tech 2003; 8(1): 4-13.

[28] Britton B, Tesser A. Effects of prior knowledge on use of cognitive capacity in three complex cognitive tasks. J Verb Learn Verb Be 1982; 21: 421-36.

[29] Brünken R, Plass JL, Leutner D. Direct measurement of cognitive load in multimedia learning. Educ Psychol 2003; 38: 53-61.

[30] Kellog RT. Effects of topic knowledge on the allocation of processing time and cognitive effort to writing processes. Mem Cogn 1987; 15(3): 256-66.

[31] Brünken R, Plass JL, Leutner D. Assessment of cognitive load in multimedia learning with dual-task methodology: Auditory load and modality effects. Instr Sci 2004; 32: 115-32.

[32] DeLeeuw K, Mayer R. A comparison of three measures of cognitive load: Evidence for separable measures of intrinsic, extraneous and germane load. J Educ Psychol 2008; 100(1): 22334.

[33] De Bruijn D, De Mul S, Van Oostendorp H. The influence of screen size and text layout on the study of text. Behav Inform Technol 1992; 11: 71-8.

[34] Paas F, Tuovinen J, Tabbers H, Van Gerven PWM. Cognitive load measurement as a means to advance Cognitive Load Theory. Educ Psychol 2003; 38(1): 63-71.

[35] Just MA, Carpenter PA. A capacity theory of comprehension: Individual differences in working memory. Psychol Rev 1992; 99: 122-49.

[36] Hochheiser H, Shneiderman B. Performance benefits of simultaneous over sequential menus as task complexity increases. Int J Hum-Comput Int 2000; 12(2): 173-92. 
[37] Kintsch W. Text comprehension, memory and learning. Am Psychol 1994; 49(4): 294-303.

[38] Anderson RC. Allocation of attention during reading. In: Flammer A, Kintsch W, Eds. Discourse processing. New York: North Holland 1982; pp. 287-99.

[39] Reynolds RE, Shirey LL. The role of attention in studying and learning. In: Goetz ET, Weinstein CE, Alexander P, Eds. Learning and Study Strategies: Issues in Assessment, Instruction and Evaluation. Washington: Academic Press 1988; pp. 77-100.
[40] Shapiro AM, Niederhauser D. Learning from hypertext: research issues and findings. In: Jonassen DH, Ed. Handbook of research on educational communications and technology. 2nd ed. Mahwah, NJ: Lawrence Erlbaum Associates 2004; pp. 605-20.

[41] Sawicka A. Dynamics of cognitive load theory: A model-based approach. Comput Hum Behav 2008; 24(3): 1041-66.

[42] McNamara DS, Shapiro AM. Multimedia and hypermedia solutions for promoting metacognitive engagement, coherence, and learning. J Educ Comput Res 2005; 33: 1-29.

Received: January 1, 2008

Revised: September 1,2008

Accepted: November 1, 2008

(C) Madrid and Cañas; Licensee Bentham Open.

This is an open access article licensed under the terms of the Creative Commons Attribution Non-Commercial License (http: //creativecommons.org/licenses/by$\mathrm{nc} / 3.0 /$ ) which permits unrestricted, non-commercial use, distribution and reproduction in any medium, provided the work is properly cited. 